\title{
A Novel Configuration Method of Virtual Machine Fitness
}

\author{
Wang Xin ${ }^{1}$ and Wang Ning ${ }^{2}$ \\ 1. Wuxi Institute of Technology, Wuxi, Jiangsu, 214121, China \\ 2. Yellow River Conservancy Technical Institute, Kaifeng, Henan,475000,China \\ E-mail:yscls2013@163.com
}

\begin{abstract}
Virtual machine resource allocation problem is closely related to the resource requirement of physical host, and a novel configuration method of virtual machine is proposed. First, the system analyzes the resource requirements of the related virtual machine to the physical host. Secondly, with the help of the similarity between the virtual machine, the fitness is configurated. Secondly, based on the degree of adaptation, the scheduling of the related virtual machine is completed. Two typical methods are selected and compared with the method in this paper. The experimental results confirm the effectiveness of the new method.
\end{abstract}

Keywords: Resource allocation, physical host, virtual machine, load

\section{Introduction}

With the increasing amount of information processing data, a lot of computing tasks can not be completed on the local computer. In this context, cloud computing as a third party computing service platform has been favored by the majority of users[1]. By integrating the hardware resources of each processor in the cloud, cloud computing can get more powerful computing power, and can complete the computing tasks with a larger amount of data[2].

At the beginning of the development of cloud computing, each specific service task is corresponding to the actual calculation of the physical host, the flexibility of this kind of tight coupling method is poor, and it is easy to cause the idle and waste of computing resources[3] . In order to realize the loose coupling of cloud services and physical hosts, the virtual technology is established. Specific tasks of cloud computing was first assigned to virtual machine, and then ccording to the configuration of the hardware resources of the physical host, the virtual machine resource is scheduled to the physical host, thus greatly enhance the flexibility of cloud computing services, and the resource utilization of computing terminal is also improved[4].

At present, the research on the virtual machine resource scheduling in cloud computing is very rich. Based on the particle swarm optimization algorithm, Jayasinghe constructed a virtual machine scheduling algorithm based on particle swarm optimization, and obtained the expected scheduling results[5]. Calheiros introduced the thermodynamic evolution algorithm from the field of thermodynamics into the virtual machine resource scheduling, and achieve a stable and reliable virtual machine allocation[6]. Hage divides the whole cloud into two-dimensional and three-dimensional structure, and constructs a virtual machine resource scheduling algorithm which is suitable for the reasonable allocation of candidate tasks though the regional block compensation mechanism[7]. Gupta pointed out that in the process of virtual machine resource scheduling, in cloud computing the topology structure of each computing terminal has an important influence. According to this, he constructed a scheduling algorithm for the virtual machine resource topology [8]. According to Bhutani, the virtual machine scheduling problem in cloud 
computing is essentially a resource optimization problem. In this way, a virtual machine resource scheduling algorithm based on $\mathrm{K}$ path decision tree is constructed[9]. According to the virtual machine scheduling process of a variety of attributes from the system decision-making theory, Cerroni constructed a multi attribute decision scheduling algorithm, and achieved an efficient virtual machine scheduling[10]. Stanik believes that energy consumption is an important goal of optimal scheduling in the process of virtual machine scheduling. For this reason, he designed a virtual machine scheduling algorithm based on the proportion of energy consumption and achieved the desired objective[11]. Rana also constructed a virtual machine scheduling method based on multi attribute decision making,and use the strategy of hierarchical analysis in the process of scheduling[12].

In the cloud computing services of many large-scale computing tasks, the virtual machines are often clustered. Which virtual machine cluster is more suitable for the current computing tasks to become a new topic in the field of virtual machine scheduling. In this paper, based on the existing algorithms of virtual machine scheduling, this paper focuses on the research of virtual machine cluster scheduling problem, and constructs a virtual machine cluster scheduling method based on genetic algorithm.

\section{Virtual Machine Cluster Scheduling Process Design}

\subsection{Virtual Machine Cluster}

The so-called virtual machine cluster, is relative to a single virtual machine. In the early phase of cloud computing services, computing tasks and hardware resources are configured and connected via a single virtual machine. With the increasing amount of computation, more virtual machines are needed to accomplish one task. Because of the consistency of the final goal, these virtual machines become an interconnected set and hence are called virtual machine clusters.

A typical virtual machine cluster is just shown as figure.1.

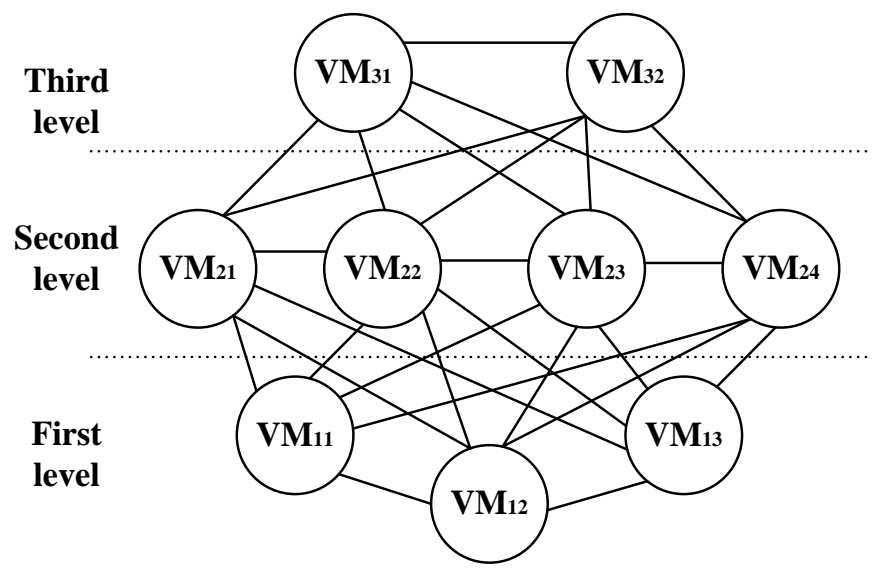

Figure 1. Virtual Machine Cluster

Virtual machine cluster scheduling problem is a new difficulty in the field of cloud computing. First, this scheduling must ensure that each virtual machine can find the appropriate physical host; secondly, the relevance of the virtual machine after scheduling also needs to be guaranteed. Of course, the premise of these two works, is how to find a virtual machine cluster suitable for the current computing tasks from the cloud of virtual machine resources. 


\subsection{Population Setting and Evolution}

Genetic algorithm is the core algorithm to solve the virtual machine cluster scheduling problem. Therefore, first of all, the virtual machine scheduling problem to form a genetic algorithm based on the description of the problem, Therefore, the first to do is to describe the problem of virtual machine scheduling based on genetic algorithm, which involves the virtual machine scheduling problem of population settings. If the cloud at the same time to deal with the calculation of a total of 20 tasks, it is better to select 4 virtual machines to be responsible for these 20 tasks. In this way, the final screen out of the 4 virtual machines is a cluster of virtual machine. In this cluster, each task is assigned to a specific virtual machine, a set of configuration of the 20 tasks constitutes a chromosome.

Assuming that $V_{1}, V_{2}, V_{3}, V_{4}$ respectively, representing 4 virtual machines, then a possible chromosome is as follows:

$$
\begin{aligned}
& D=\left[V_{4}, V_{3}, V_{1}, V_{2},\right. V_{2}, \\
& V_{4}, V_{3}, V_{4}, V_{2}, V_{1}, \\
& V_{1}, V_{1}, V_{2}, V_{3}, V_{4}, \\
&\left.V_{1}, V_{3}, V_{2}, V_{4}, V_{4}\right]
\end{aligned}
$$

The chromosome configuration showed that model $V_{1}$ virtual machine is responsible for the task are No. 3, 10,11, 12, 16; $V_{2}$ is responsible for the task are No.4, 5, 9, 13, 18; $V_{3}$ is responsible for the task are No. 2, 7, 14, 17; $V_{4}$ is responsible for the task are No.1, $6,8,15,19$ and 20 .

The genetic algorithm is used to accomplish the scheduling of virtual machine cluster, which can be used to deal with the possible genetic iterative process. Finally, according to the actual situation of the virtual machine resource allocation, the optimal chromosome is determined.

According to the formula (1), 10 chromosomes were constructed as the initial population of genetic algorithm, and then continuously derive new population individuals according to the selection, crossover and mutation operation of genetic algorithm.

\subsection{Adaptive Function Design}

In the genetic algorithm, the iteration process is based on the judgment of the adaptive function, and the adaptive function is designed according to the demand of the genetic evolution. After all the iterative process, the best chromosome which determined by the adaptive degree function will be finalized. For this study, when the best chromosome is determined, which virtual machine cluster is selected by the cloud, which virtual machine is responsible for which task is determined.

We use $\hat{V}$ to represent the virtual machine cluster, which can be described in the following mathematical form:

$$
\hat{V}=\left\{V_{1}, V_{2}, \cdots, V_{i}, \cdots\right\}
$$

Using $\hat{R}$ to represent the task set which is to be completed, This collection can be described in the following mathematical form:

$$
\hat{R}=\left\{R_{1}, R_{2}, \cdots, R_{j}, \cdots\right\}
$$


Virtual machine is responsible for the implementation of computing tasks, making the most efficient use of virtual machine resources is an important criterion. In addition, the calculation time and service price of the virtual machine are also important indicators need to be considered. For this purpose, this paper will construct the adaptive function from three aspects.

From the point of view of resource utilization, the most important resources in the process of cloud computing services include CPU, memory and communication bandwidth. According to this, we construct the virtual machine scheduling adaptive function from the angle of resource utilization as follows:

$$
S_{1}=w_{C P U}\left(\frac{\sum_{j=1}^{J} R_{j}^{F_{C P U}}}{\sum_{i=1}^{I} V_{i}^{F_{C P U}}}+\frac{\sum_{j=1}^{J} R_{j}^{C_{C P U}}}{\sum_{j=1}^{J} V_{j}^{C_{C P U}}}\right)+w_{R A M} \frac{\sum_{j=1}^{J} R_{j}^{R A M}}{\sum_{j=1}^{J} V_{j}^{R A M}}+w_{B W} \frac{\sum_{j=1}^{J} R_{j}^{B W}}{\sum_{j=1}^{J} V_{j}^{B W}}
$$

In above calculation formula, $R_{j}^{F_{C P U}}$ denotes the $\mathrm{j}$-th computing task CPU main frequency demand, $R_{j}^{C_{C P U}}$ denotes the $\mathrm{j}$-th computing task CPU Kernel number demand, $R_{j}^{R A M}$ denotes the $\mathrm{j}$-th computing task memory size requirements, $R_{j}^{R A M}$ denotes the $\mathrm{j}$-th computing task communication bandwidth demand; $R_{j}^{B W}$ denotes the i-th a virtual machine CPU frequency size, $V_{i}^{F_{C P U}}$ denotes the i-th a virtual machine CPU Kernel number size, $V_{i}^{R A M}$ denotes the i-th a virtual machine memory size, $V_{j}^{B W}$ denotes the i-th a virtual machine communication bandwidth demand; $w_{C P U}, w_{R A M}, w_{B W}$, respectively, denotes CPU utilization efficiency, memory utilization efficiency, communication bandwidth utilization efficiency whose weights in $S_{1}$.

If we look at the calculation time alone, undoubtedly, the configuration scheme which cost the least computation time is the best. Assuming that all virtual machines are beginning to perform the calculation task at same time, then the last time to complete the task of the virtual machine is the least computing time of cluster virtual machine, according to the above inference, the adaptive function of this design is as follows:

$$
S_{2}=\max _{I}\left\{T_{V_{1}}, T_{V_{2}}, \cdots, T_{V_{i}}, \cdots\right\}
$$

In above calculation formula, $T_{V_{i}}$ represents the time required for the i-th virtual machine to complete all of its computing tasks.

If we look at the service price alone, no doubt, the lowest price of the service configuration is the best solution, according to the above inference, the design of the adaptive subfunction is as follows:

$$
S_{3}=\min _{L}\left\{\sum_{i}^{I} C_{V_{1}}\right\}
$$

In above calculation formula, $C_{V_{i}}$ represents the demand of computed value that the ith virtual machine to complete its own computing tasks required in a scheme, and $\mathrm{L}$ represents all possible cluster solutions. 
From the impact trend of the three adaptive function on the overall adaptive function, $S_{1}$ has a positive impact, $S_{2}$ and $S_{3}$ will have a negative impact, so the total adaptive function constructed is as follows:

$$
S=\pi_{1} S_{1}-\pi_{2} S_{2}-\pi_{3} S_{3}
$$

In above calculation formula, $\pi_{1}, \pi_{2}, \pi_{3}$ respectively expressed $S_{1}, S_{2}, S_{3}$ whose impact on the weight of $S$.

So far, the construction of virtual machine cluster resource scheduling adaptation degree function has been completed. In every rounds of iterative process, we will rely on the adaptive function to select the optimal scheduling scheme. In the genetic algorithm, we need to based on the probability of each chromosome was selected in the all round to select the best chromosome from each chromosome. For this purpose, we first calculate the probability of that $D_{i}$ is selected in the k-th iteration, as shown in the formula (8).

$$
p_{k}\left(D_{i}\right)=\frac{S^{k}\left(D_{i}\right)}{\sum_{i=1}^{I} S^{k}\left(D_{i}\right)}
$$

Accordingly, we can calculate the cumulative probability of chromosome $D_{i}$ was selected in the all round, as shown in formula (9).

$$
p\left(D_{i}\right)=\sum_{k=1}^{K} p_{k}\left(D_{i}\right)
$$

In the whole iterative process, the largest chromosome of $p\left(D_{i}\right)$ will be inherited, which corresponds to the optimal scheduling scheme of cluster virtual machine.

\section{Experimental Results and Analysis of Virtual Machine Cluster Scheduling}

In front, we describe the cluster virtual machine resource scheduling algorithm based on genetic algorithm in detail. The performance of this method is verified by experiments below.

In the experiment, we chose CloudSim as the simulation experiment platform to test the performance of the algorithm. In order to realize the horizontal alignment of the scheduling method, we also select the virtual machine scheduling method based on load balancing, and the virtual machine scheduling method based on resource balance as the analogy algorithm.

For the purpose to examine this three scheduling algorithm scheduling capabilities for different number of virtual machines, we set the maximum number of virtual machines as 40 on the CloudSim platform, and gradually increase the number of virtual machines involved in scheduling. In all the 40 virtual machines, the minimum CPU core is one and the maximum is four. The virtual machine CPU frequency is $1000 \mathrm{GHz}$ to $2500 \mathrm{GHz}$, and the memory size is $1 \mathrm{~GB}$ to $8 \mathrm{~GB}$. The virtual machine communication bandwidth is set $100 \mathrm{MB} / \mathrm{S}$ to $1000 \mathrm{MB} / \mathrm{S}$, and the lowest price of the virtual machine service is $20 \mathrm{RMB} /$ Min up to 100RMB / Min. 
In this experiment, there are 20 computational tasks required to be completed, and the requirements for CPU, memory and communication bandwidth are as shown in Table 1 in this 20 tasks.

Table 1. The Rresource Requirements for the 20 Tasks

\begin{tabular}{|c|c|c|c|c|}
\hline Task & Frequency & Core & Memory & Bandwidth \\
\hline 1 & 100 (MIPS) & Single core & $100 \mathrm{MB}$ & $10(\mathrm{MB} / \mathrm{s})$ \\
\hline 2 & 100 (MIPS) & Single core & $100 \mathrm{MB}$ & $20(\mathrm{MB} / \mathrm{s})$ \\
\hline 3 & 100 (MIPS) & Single core & $200 \mathrm{MB}$ & $20(\mathrm{MB} / \mathrm{s})$ \\
\hline 4 & 100 (MIPS) & Single core & $200 \mathrm{MB}$ & $30(\mathrm{MB} / \mathrm{s})$ \\
\hline 5 & 100 (MIPS) & Single core & $300 \mathrm{MB}$ & $30(\mathrm{MB} / \mathrm{s})$ \\
\hline 6 & 200 (MIPS) & Single core & $200 \mathrm{MB}$ & $20(\mathrm{MB} / \mathrm{s})$ \\
\hline 7 & 200 (MIPS) & Single core & $300 \mathrm{MB}$ & $20(\mathrm{MB} / \mathrm{s})$ \\
\hline 8 & 200 (MIPS) & Single core & $300 \mathrm{MB}$ & $30(\mathrm{MB} / \mathrm{s})$ \\
\hline 9 & 200 (MIPS) & dual-core & $200 \mathrm{MB}$ & $20(\mathrm{MB} / \mathrm{s})$ \\
\hline 10 & 200 (MIPS) & dual-core & $200 \mathrm{MB}$ & $30(\mathrm{MB} / \mathrm{s})$ \\
\hline 11 & 300 (MIPS) & Single core & $300 \mathrm{MB}$ & $30(\mathrm{MB} / \mathrm{s})$ \\
\hline 12 & 300 (MIPS) & Single core & $300 \mathrm{MB}$ & $40(\mathrm{MB} / \mathrm{s})$ \\
\hline 13 & 300 (MIPS) & Single core & $400 \mathrm{MB}$ & $40(\mathrm{MB} / \mathrm{s})$ \\
\hline 14 & 300 (MIPS) & dual-core & $400 \mathrm{MB}$ & $40(\mathrm{MB} / \mathrm{s})$ \\
\hline 15 & 300 (MIPS) & dual-core & $400 \mathrm{MB}$ & $50(\mathrm{MB} / \mathrm{s})$ \\
\hline 16 & 400 (MIPS) & Single core & $400 \mathrm{MB}$ & $40(\mathrm{MB} / \mathrm{s})$ \\
\hline 17 & 400 (MIPS) & Single core & $500 \mathrm{MB}$ & $40(\mathrm{MB} / \mathrm{s})$ \\
\hline 18 & 400 (MIPS) & Single core & $500 \mathrm{MB}$ & $50(\mathrm{MB} / \mathrm{s})$ \\
\hline 19 & 400 (MIPS) & dual-core & $500 \mathrm{MB}$ & $50(\mathrm{MB} / \mathrm{s})$ \\
\hline 20 & 400 (MIPS) & dual-core & $600 \mathrm{MB}$ & $50(\mathrm{MB} / \mathrm{s})$ \\
\hline
\end{tabular}

For the method constructed in this paper, the key parameters in the experiment are given as follows: $w_{B W}, w_{R A M}$ and $w_{B W}$ are $0.4,0.3$ and 0.3 , respectively; $\pi_{1}, \pi_{2}$ and $\pi_{3}$ are $0.5,0.4,0.1$, respectively.From this configuration, we should highlight the impact of technical requirements on scheduling as much as possible, weakening the impact of service costs. 2.

Three methods to form the resource utilization ratio after scheduling is shown in figure 


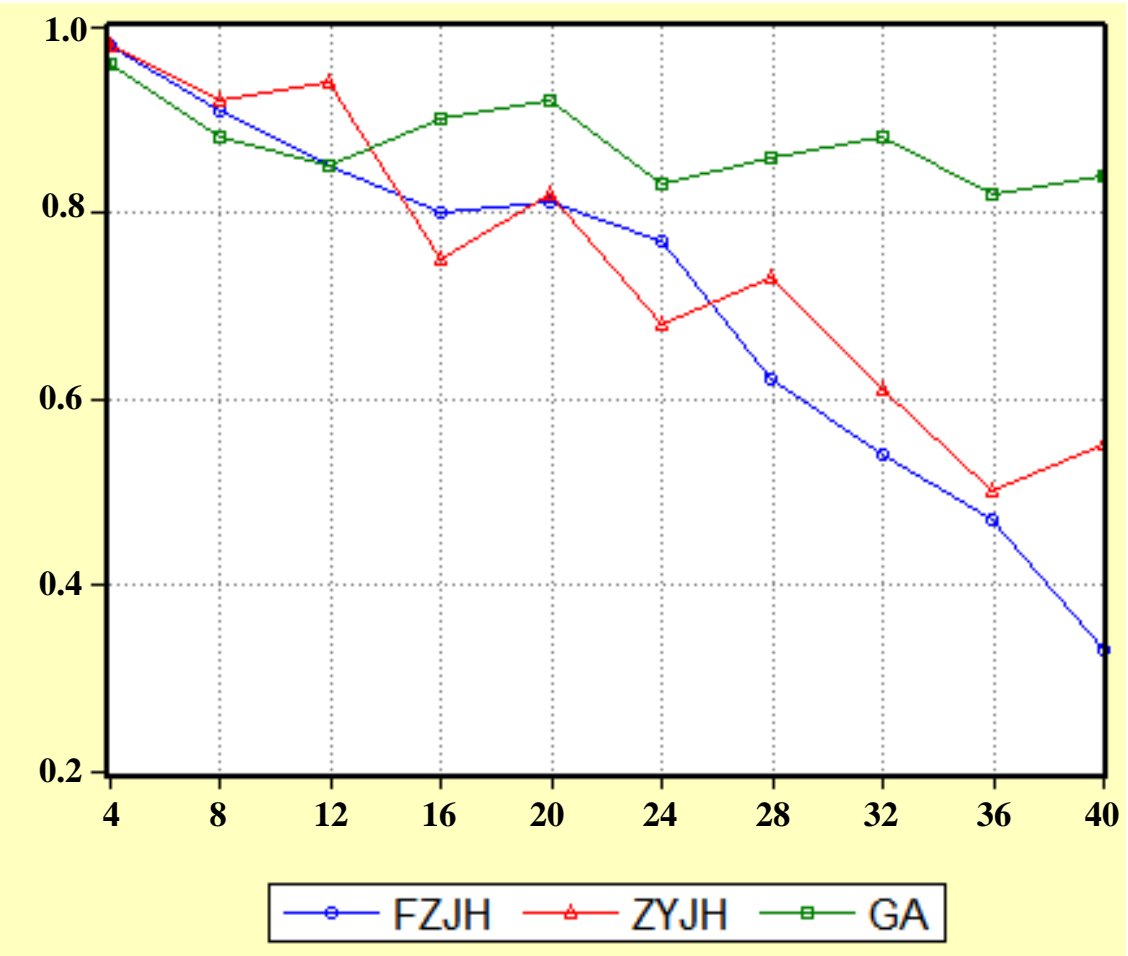

\section{Figure 2. Comparison of Resource Utilization after Scheduling in Three Ways}

In Figure 2, the ordinate represents the resource utilization, and the abscissa represents the number of virtual machines in the scheduling. FZJH represents the load balancing scheduling method, ZYJH represents the resource balancing scheduling method, and GA represents the scheduling method based on genetic algorithm proposed in this paper.

From the curve of Figure 2, we can see that when the number of virtual machines is relatively small, the utilization of the three methods are relatively high. However, with the increasing number of virtual machines participating in scheduling, the method of this paper shows the advantage, which has been stable at more than 0.8 of the resource utilization. 3.

Below, the scheduling time of the three methods is further compared as shown in figure 


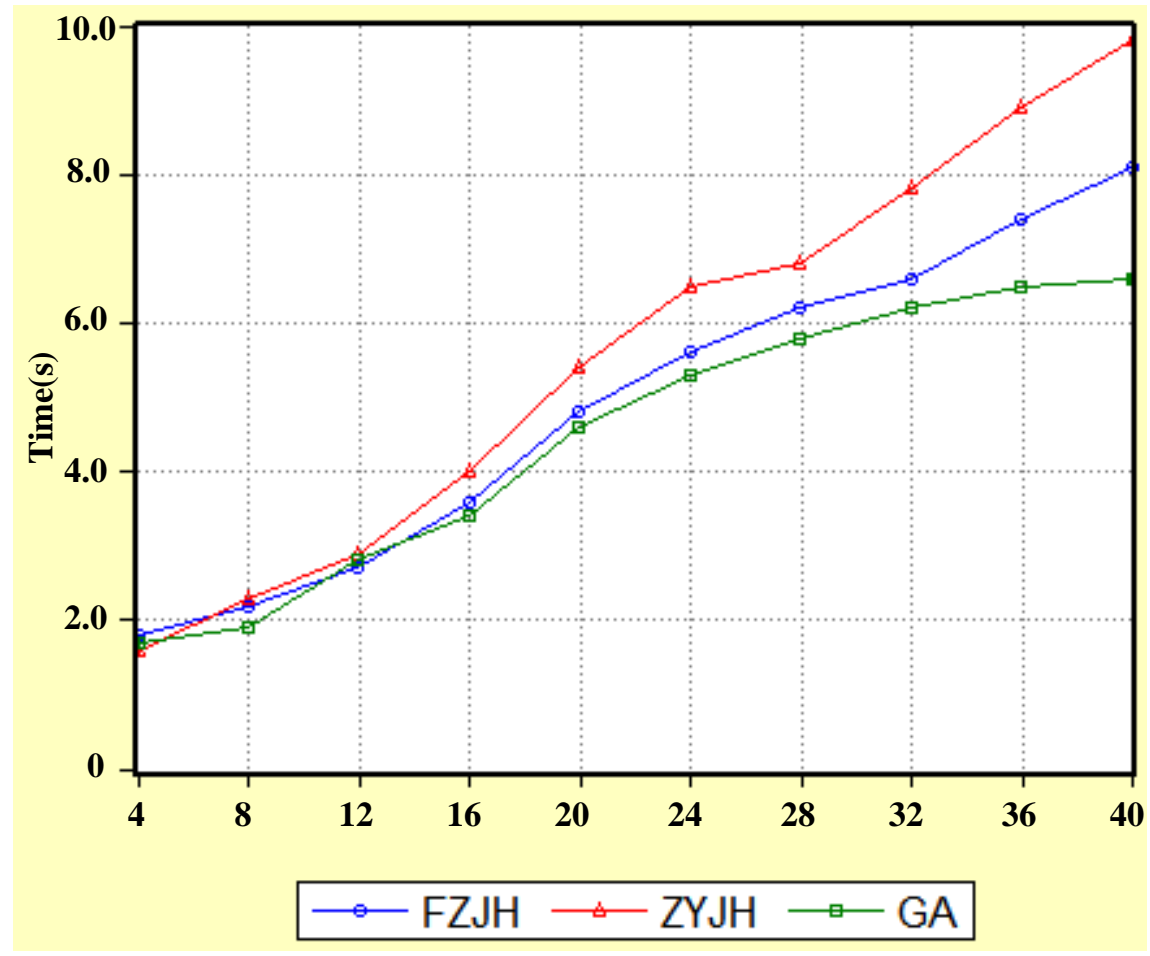

Figure 3. Comparison of Scheduling Time of Three Methods

In Figure 3, the ordinate represents the scheduling time, and the abscissa represents the number of virtual machines that participate in the scheduling.

From Figure 3, we can see that with the increase of the number of virtual machines involved in scheduling, the scheduling time of the three methods are increasing, the scheduling method based on resource balance is most obvious. Based on genetic algorithm, this paper proposes a cluster virtual machine resource scheduling method, and the scheduling time is relatively slow.

Above two groups of experiments, it fully shows the advantages of resource utilization and execution time of virtual machine cluster resource scheduling based on genetic algorithm, so as to prove the effectiveness of this method in the virtual machine cluster scheduling.

\section{Conclusion}

Big data in the cloud service request, makes the cloud computing virtualization technology from single virtual machine scheduling to cluster virtual machine scheduling. A scheduling strategy based on genetic algorithm is proposed to satisfy the scheduling process of cluster virtual machine. In the proposed scheduling strategy, the initial population of virtual machine is carried out and the chromosome is constructed. Secondly, the selection, crossover and mutation operation in genetic process is set up. Finally, according to the requirements of CPU, memory, bandwidth and other resources, the fitness function of the genetic process is constructed. Two sets of experimental results confirm that the proposed scheduling strategy is suitable for scheduling problems with cluster virtual machines. 


\section{Acknowledgements}

This study was supported by National Natural Science Foundation of China under Grant No.61502204 (Name: Research on the load balancing technology for scalable switch fabric) and a Project Funded by the Excellent Specialties Program Development of Jiangsu Higher Education Institutions(PESP) No.PPZY2015C240.

This study was supported by Key Scientific Research Projects of Henan Province University(15A520023).

\section{References}

[1] Esfandiarpoor Sina, Pahlavan Ali, Goudarzi Maziar. Structure-aware online virtual machine consolidation for datacenter energy improvement in cloud computing $[\mathrm{J}]$. Computers and Electrical Engineering, 42: 74-89(2015).

[2] Beloglazov Anton, Buyya rajkumar. OpenStack Neat: a framework for dynamic and energy-effient consolidation of virtual machines in OpenStack clouds[J]. Concurrency Computation, 27(5): 13101333(2015).

[3] Armbrust M, Fox A, Griffith R. Joseph A.D, Katz R, Konwinski A, Lee G, Patterson G.. A view of cloud computing $[\mathrm{J}]$. Communications of the ACM, 53(4): 50-58 (2010).

[4] Al-Said Taimur, Rana Omer, Burnap Peter. VMInformant: an instrumented virtual machine to support trustworthy cloud computing[J]. International Journal of High Performance Computing and Networking, 8(3): 222-234(2015).

[5] Jayasinghe D, Pu C, Eilam T. Improving performance and availability of services hosted on IaaS clouds with structural constraint-aware virtual machine placement. 2011 IEEE International Conference on Services Computing. Piscataway: IEEE, $72-79$ (2011).

[6] Calheiros R.N. and et al. CloudSim: a toolkit for modeling and simulation of cloud computing environments and evaluation of resource provisioning algorithms[J]. Software: practice and experience, 41(1): 23-50 (2011).

[7] Hage Thomas, Begnum Kyrre, Yazidi Anis. Saving the planet with bin packing-experineces using 2D and 3D bin packing of virtual machines for greener clouds[C]. Proceedings of the International Conference on Cloud Computing Technology and Science CloudCom, 240-245(2012).

[8] Gupta R, Bose S.K., Sundarrajan S. A two stage heuristic algorithm for solving the server consolidation problem with item-item and bin-item incompatibility constraints[C]. SCC 08: Proceedings of the 2008 IEEE International Conference on Services Computing. Piscataway: IEEE, 39-46 (2008).

[9] Bhutani Akshi, Jauhari Isha, Kaushik Vinay Kumar. Optimized virtual machine tree based scheduling technique in cloud using K-way trees[C]. Proceedings International Confrence on Cognitive Computing and Information Processing, 1200-1211(2015).

[10] Cerroni Walter. Network performance of multiple virtual machine live migration in cloud federations[J]. Journal of Internet Services and Applications, 6(1): 234-241(2015).

[11] A.Stanik M, HovestadtO Kao. Hardware as a service (HaaS): the completion of the cloud stack[C]. $8^{\text {th }}$ International Conference on Computing Technology and Information Management, 830-835, (2012).

[12] Rana S, Jasola S, Kumar R. A hybrid sequential approach for data clustering using K-means and particle swarm optimization algorithm[J]. International Journal of Engineering, Science and Technology, 2(6): 167-176 (2012). 
International Journal of Grid and Distributed Computing Vol. 9, No. 11 (2016) 